\title{
On Hamilton-Connectivity and Detour Index of Certain Families of Convex Polytopes
}

\author{
Sakander Hayat $\left(\mathbb{D},{ }^{1}\right.$ Muhammad Yasir Hayat Malik $\left(\mathbb{D},{ }^{2}\right.$ Ali Ahmad $\left(\mathbb{D},{ }^{3}\right.$ Suliman Khan (D), \\ Faisal Yousafzai iD, ${ }^{4}$ and Roslan Hasni iD ${ }^{5}$ \\ ${ }^{1}$ Faculty of Engineering Sciences, GIK Institute of Engineering Sciences and Technology, Topi, \\ Khyber Pakhtunkhwa 23460, Pakistan \\ ${ }^{2}$ Department of Mathematics, Government College University, Faisalabad 38000, Pakistan \\ ${ }^{3}$ College of Computer Science and Information Technology, Jazan University, Jazan 45142, Saudi Arabia \\ ${ }^{4}$ Military College of Engineering, National University of Sciences and Technology (NUST), Islamabad 44000, Pakistan \\ ${ }^{5}$ School of Informatics and Applied Mathematics, University Malaysia Terengganu, Kuala Terengganu, \\ Terengganu 21030, Malaysia
}

Correspondence should be addressed to Sakander Hayat; sakander1566@gmail.com

Received 11 February 2021; Revised 21 June 2021; Accepted 8 July 2021; Published 19 July 2021

Academic Editor: Abdul Qadeer Khan

Copyright (c) 2021 Sakander Hayat et al. This is an open access article distributed under the Creative Commons Attribution License, which permits unrestricted use, distribution, and reproduction in any medium, provided the original work is properly cited.

\begin{abstract}
A convex polytope is the convex hull of a finite set of points in the Euclidean space $\mathbb{R}^{n}$. By preserving the adjacency-incidence relation between vertices of a polytope, its structural graph is constructed. A graph is called Hamilton-connected if there exists at least one Hamiltonian path between any of its two vertices. The detour index is defined to be the sum of the lengths of longest distances, i.e., detours between vertices in a graph. Hamiltonian and Hamilton-connected graphs have diverse applications in computer science and electrical engineering, whereas the detour index has important applications in chemistry. Checking whether a graph is Hamilton-connected and computing the detour index of an arbitrary graph are both NP-complete problems. In this paper, we study these problems simultaneously for certain families of convex polytopes. We construct two infinite families of Hamilton-connected convex polytopes. Hamilton-connectivity is shown by constructing Hamiltonian paths between any pair of vertices. We then use the Hamilton-connectivity to compute the detour index of these families. A family of non-Hamiltonconnected convex polytopes has also been constructed to show that not all convex polytope families are Hamilton-connected.
\end{abstract}

\section{Introduction and Preliminaries}

All graphs in this paper are simple, loopless, finite, and connected.

A graph $G$ is an ordered pair $G=(V(G), E(G))$ with $V(G)$ as its vertex set (i.e., set of points called vertices) and $E(G) \subseteq\left(\begin{array}{c}V(G) \\ 2\end{array}\right)$ as its edge set (i.e., set of lines connecting points called edges). The number of vertices, say $n:=|V(G)|$, is called the order of $G$ and the number of edges, say $m:=|E(G)|$, is called the size of $G$. For two vertices $x, y \in V(G)$, we write $x \sim y$ if both $x$ and $y$ are adjacent, i.e., they are connected by an edge. For $U \subseteq V(G)$ and $\quad x, y \in V(G), \quad$ if $\quad U=\left\{u_{i}: 1 \leq i \leq p\right\}$, then $\quad x \circ$ $\left\{u_{i}: 1 \leq i \leq p\right\} \circ y$ means that $x \sim u_{1}$ and $u_{p} \sim y$ and adjacency in the rest of $u_{i}$ 's $(2 \leq i \leq p)$ stays the same. For a positive integer $v \in \mathbb{Z}^{+}$, we write $v \mid 2$ (resp. $v \nmid 2$ ) if $v$ is even (resp. odd).

A Hamiltonian cycle $C_{H}(x)$ in a connected graph $G$ starting and finishing at the vertex $x$ is a cycle traversing all the vertices of $G$. Similarly, a Hamiltonian path $P_{H}(x, y)$ between vertices $x$ and $y$ is the one covering the entire graph without missing any vertex. A graph comprising a Hamiltonian path (resp. Hamiltonian cycle) is called traceable (resp. Hamiltonian). Every Hamiltonian graph, by definition, is traceable, whereas the converse is not true in general. 
For instance, the so-called Petersen graph does not contain any Hamiltonian cycle and, thus, is not Hamiltonian. However, you can easily find a Hamiltonian path between two of its vertices, which makes it traceable but not Hamiltonian. Graphs comprising Hamiltonian paths between every pair of its vertices are called Hamilton-connected. They were introduced and studied in 1963 by Ore [1]. Trivalent Hamiltonian graphs and their canonical representation were studied by Frucht [2]. Hamilton-connectivity and Hamiltoniancity possess some extensive available literature. See, for example, [3-7].

By preserving the vertex-edge incidence relation in convex polytopes, their graphs are constructed. Bača [8-10] was among the first researchers to consider these families of geometric graphs. In [10] (resp. [9]), Bača studied the problem of magic (resp. graceful and antigraceful) labeling of convex polytopes, whereas in [8], the problem of face antimagic labeling of convex polytopes was studied. Miller et al. [11] studied the vertex-magic total labeling of convex polytopes. Imran et al. [12-15] computed the minimum metric dimension of various infinite families of convex polytopes. In particular, they showed that these infinite families of convex polytopes have constant metric dimension. Malik and Sarwar [16] also constructed two infinite families of convex polytopes having constant metric dimension. Other closely related infinite families of graphs with constant metric dimension are studied in [17]. Kratica et al. [18] studied the strong metric dimension of certain infinite families of convex polytopes by constructing their doubly resolving sets. The fault-tolerant metric dimension (resp. mixed metric dimension) of convex polytopes was studied by Raza et al. [19] (resp. Raza et al. [20]). The binary locating-dominating number of convex polytopes is studied by Simić et al. [21] and Raza et al. [22]. The open-locatingdominating number of certain convex polytopes has recently been studied by Savić et al. [23]. Hayat et al. [24] studied Hamilton-connectivity and detour index in convex polytopes.

For a graph $G$, let $\ell(x, y)$ be the length of the longest path (i.e., detour) between vertices $x$ and $y$ of $G$. The detour index [25] is defined to be the sum of the lengths of the detour between unordered pairs of vertices in $G$. The detour index of a graph $G$ is usually denoted by $\omega(G)$.

$$
\omega(G)=\sum_{\{x, y\} \subset V(G)} \ell(x, y) .
$$

In chemistry, the detour index has diverse applications. Lukovits [26] put forward its QSAR/QSPR applications. Trinajstić et al. [27] presented some more of its chemical applications and compared its predictive potential in correlating the normal boiling points of benzenoid hydrocarbons with the performance of Wiener index. Rücker and Rücker [28] presented more of its rigorous applications for correlating the boiling points of acyclic and cyclic alkanes. The calculation of the detour index for a given graph has been shown an NP-complete problem in [29].

Mahmiani et al. [30] proposed the edge versions of the detour index and studied their mathematical properties.
Zhou and Cai [31] proved some upper and lower bounds on the detour index of graphs. Qi and Zhou [32] studied minimum uncyclic graphs with respect to the detour index. Du [33] studied the minimum detour index of bicyclic graphs. Fang et al. [34] characterized the minimum detour index of some families of tricyclic graphs. Karbasioun et al. [35] studied the applications of the detour index in infinite families of nanostar dendrimers. Wu and Deng [36] computed the detour index for a chain of C20 fullerenes. Kaladevi and Abinayaa [37] studied spectral properties of the detour index in relation with the Laplacian energy of graphs. Recently, Abdullah and Omar [38] introduced the restricted edge version of the detour index and studied it for some families of graphs. Tang et al. [39] studied Zagreb connection indices of some operation of graphs.

Let $S_{v}$ denote the $v$-dimensional star graph on $v+1$ vertices. We end this section with an important and wellknown result bounding the detour index in terms of its order.

Theorem 1 [40]. Let $G$ be an v-vertex graph with $v \geq 3$ and $\omega(G)$ be its detour index. Then,

$$
(\nu-1)^{2} \leq \omega(G) \leq \frac{\nu(\nu-1)^{2}}{2},
$$

with left equality if and only if $G \cong S_{v}$, and right inequality holds if and only if $G$ is Hamilton-connected.

\section{A Family of Non-Hamilton-Connected Convex Polytopes}

Bača [10] introduced the graph of convex polytope $D_{v}$ for $v \geq 4$. It is a family of convex polytopes comprising $2 v$ pentagonal faces. See Figure 1 for the $\nu$-dimensional family of convex polytopes $D_{v}$.

Mathematically, the vertex set of $D_{v}$ consists of four layers of vertices, i.e., $w_{p}, x_{p}, y_{p}$, and $z_{p}$. That is to say that $V\left(D_{\nu}\right)=\left\{w_{p}, x_{p}, y_{p}, z_{p}: 1 \leq p \leq v\right\}$. Accordingly, the edge set of $D_{\gamma}$ is as follows:

$$
\begin{aligned}
E\left(D_{\nu}\right) & =\left\{w_{p} w_{p+1}, z_{p} z_{p+1}, w_{p} x_{p}, x_{p} y_{p}, x_{p+1} y_{p}, y_{p} z_{p}: 1\right. \\
& \leq p \leq \nu\} .
\end{aligned}
$$

The subscripts are to be considered modulo $v$. The layer of vertices comprising $w_{p}$ is called the inner layer, whereas the layer comprising $z_{p}$ is called the outer layer of $D_{v}$. The vertices $x_{p}$ and $y_{p}, 1 \leq p \leq v$ form the middle layers.

The following result shows that the infinite family $D_{\nu}$ of convex polytopes is not Hamilton-connected.

Proposition 1. The $v$-dimensional convex polytope $D_{v}$, with $\nu \geq 4$, is non-Hamilton-connected.

Proof. It is enough to show that there exist two vertices in the $v$-dimensional convex polytope $D_{v}$ such that no Hamiltonian path exists between them. 


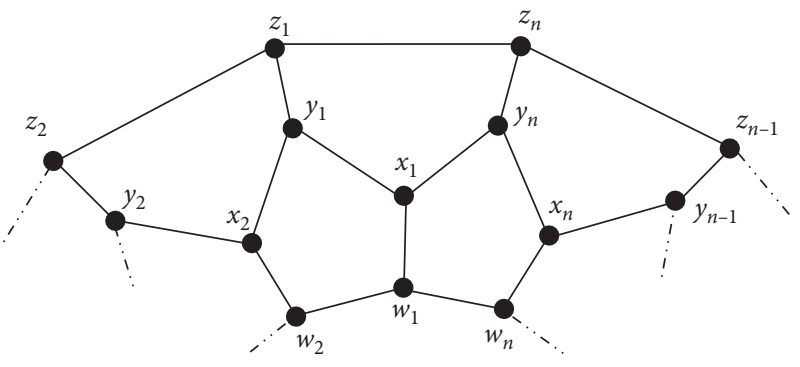

Figure 1: The graph of convex polytope $D_{v}$.

It is easy to see that, between every pair of vertices at distance two on the outer layer, i.e., between $z_{p}$ and $z_{p+2}(1 \leq p \leq v-1)$, there exists no Hamiltonian path because of the two pentagonal layers of faces. This shows that the $v$-dimensional convex polytope $D_{v}$ is non-Hamiltonconnected.

\section{Hamilton-Connectivity and the Detour Index of $H_{v}$}

In this section, we show that the graph of $v$-dimensional convex polytope $H_{v}$ is Hamilton-connected. Then, we use its Hamilton-connectivity to find a formula for its detour index. This family of convex polytopes was introduced by Imran and Siddiqui [14].

Mathematically, the vertex set of $H_{v}$ consists of four layers of vertices, i.e., $v_{p}, w_{p}, x_{p}, y_{p}$, and $z_{p}$. That is to say that $V\left(H_{v}\right)=\left\{v_{p}, w_{p}, x_{p}, y_{p}, z_{p}: 1 \leq p \leq \nu\right\}$. Accordingly, the edge set of $H_{v}$ is as follows:

$$
\begin{aligned}
E\left(H_{\nu}\right)= & \left\{v_{p} v_{p+1}, v_{p} w_{p}, w_{p} v_{p+1}, w_{p} w_{p+1}, w_{p} x_{p}, x_{p} x_{p+1},\right. \\
& \left.x_{p} y_{p}, y_{p} x_{p+1}, y_{p} y_{p+1}, y_{p} z_{p}, z_{p} z_{p+1}: 1 \leq p \leq v\right\} .
\end{aligned}
$$

The subscripts are to be considered modulo $\nu$. See Figure 2 to view the $\nu$-dimensional convex polytope graph $H_{\gamma}$.

The following is the main result of this section.

Theorem 2. The graph of $\nu$-dimensional convex polytope $H_{\nu}$, with $v \geq 5$, is Hamilton-connected.

Proof. We prove this result by definition. For this, we have to show that there exist Hamiltonian paths between any pair of vertices of $H_{v}$.

Let $P_{H}(u, v)$ be a Hamiltonian path between vertices $u$ and $v$ in $H_{v}$. Let $V\left(H_{v}\right)=Z \cup Y \cup X \cup W \cup V$ such that $Z=\left\{z_{1}, z_{2}, \ldots, z_{\nu}\right\}, \quad Y=\left\{y_{1}, y_{2}, \ldots, y_{\nu}\right\}, X=\left\{x_{1}, x_{2}, \ldots\right.$, $\left.x_{\nu}\right\}, W=\left\{w_{1}, w_{2}, \ldots, w_{\nu}\right\}$, and $V=\left\{v_{1}, v_{2}, \ldots, v_{\nu}\right\}$ (see Figure 2).

Case 1: $u^{\prime}=z_{1}$ and $v^{\prime}=z_{p}, 2 \leq p \leq v$

Subcase 1.1: $2 \leq p \leq v-2$ :

$$
\begin{aligned}
P_{H}\left(u^{\prime}, v^{\prime}\right): u^{\prime}= & z_{1} \circ\left\{z_{v-q}: 0 \leq q \leq v-p-1\right\} \circ\left\{y_{p-q+1}: 0 \leq q \leq p-2\right\} \circ\left\{x_{q}: 3 \leq q \leq p+1\right\} \circ \\
& \left\{x_{q} y_{q}: p+2 \leq q \leq v\right\} \circ y_{1} x_{1} w_{1} \circ\left\{v_{q}: 1 \leq q \leq v\right\} \circ\left\{w_{v-q}: 0 \leq q \leq v-2\right\} \circ x_{2} y_{2} \circ\left\{z_{q}: 2 \leq q \leq p\right\}=v^{\prime} .
\end{aligned}
$$

Subcase 1.2: $p=v-1$ :

$$
\begin{aligned}
P_{H}\left(u^{\prime}, v^{\prime}\right): u^{\prime}= & z_{1} z_{v} y_{v} y_{1} x_{1} w_{1} \circ\left\{y_{q}: 1 \leq q \leq v\right\} \circ\left\{w_{v-q}: 0 \leq q \leq v-2\right\} \circ \\
& \left\{x_{q}: 2 \leq q \leq v\right\} \circ\left\{y_{v-q}: 1 \leq q \leq v-2\right\} \circ\left\{z_{q}: 2 \leq q \leq v-1\right\}=v^{\prime} .
\end{aligned}
$$

Subcase 1.3: $p=v$ :

$$
\begin{aligned}
P_{H}\left(u^{\prime}, v^{\prime}\right): u^{\prime}= & z_{1} y_{1} x_{1} w_{1} \circ\left\{v_{q}: 1 \leq q \leq \nu\right\} \circ\left\{w_{\nu-q}: 0 \leq q \leq \nu-2\right\} \circ \\
& \left\{x_{q}: 2 \leq q \leq \nu\right\} \circ\left\{y_{\nu-q}: 0 \leq q \leq v-2\right\} \circ\left\{z_{q}: 2 \leq q \leq \nu\right\}=v^{\prime} .
\end{aligned}
$$

Case 2: $u^{\prime}=z_{1}$ and $v^{\prime}=y_{p}, 1 \leq p \leq v$

Subcase 2.1: $1 \leq p \leq v-1$ : 


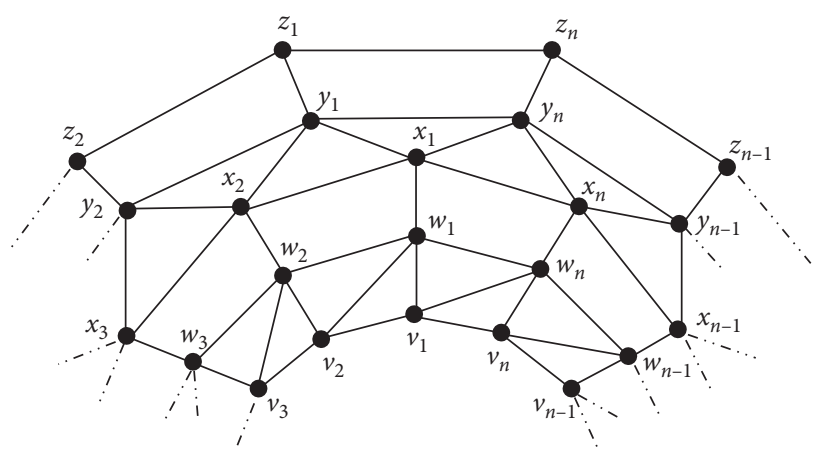

FIgURE 2: The $v$-dimensional convex polytope $H_{\nu}$.

$$
\begin{aligned}
P_{H}\left(u^{\prime}, v^{\prime}\right): u^{\prime}= & \left\{z_{q}: 1 \leq q \leq \nu\right\} \circ\left\{y_{\nu-q}: 0 \leq q \leq \nu-p-1\right\} \circ\left\{x_{q}: p+1 \leq q \leq \nu\right\} \circ \\
& \left\{w_{\nu-q}: 0 \leq q \leq \nu-2\right\} \circ\left\{v_{q}: 2 \leq q \leq \nu\right\} \circ v_{1} w_{1} \circ\left\{x_{q} y_{q}: 1 \leq q \leq p\right\}=v^{\prime} .
\end{aligned}
$$

Subcase 2.2: $p=v$ :

$$
\begin{aligned}
P_{H}\left(u^{\prime}, v^{\prime}\right): u^{\prime}= & z_{1} \circ\left\{z_{\nu-q}: 0 \leq q \leq \nu-2\right\} \circ\left\{y_{q}: 2 \leq q \leq \nu-1\right\} \circ \\
& \left\{x_{v-q}: 0 \leq q \leq v-2\right\} \circ\left\{w_{q}: 2 \leq q \leq \nu\right\} \circ\left\{v_{v-q}: 0 \leq q \leq \nu-1\right\} \circ w_{1} x_{1} y_{1} y_{\nu}=v^{\prime} .
\end{aligned}
$$

Case 3: $u^{\prime}=z_{1}$ and $v^{\prime}=x_{p}, 1 \leq p \leq v$

Subcase 3.1: $1 \leq p \leq \nu-1$ :

$$
\begin{aligned}
P_{H}\left(u^{\prime}, v^{\prime}\right): u^{\prime}= & \left\{z_{q}: 1 \leq q \leq \nu\right\} \circ\left\{y_{\nu-q}: 0 \leq q \leq v-p\right\} \circ\left\{x_{q}: p+1 \leq q \leq \nu\right\} \circ \\
& \left\{w_{\nu-q}: 0 \leq q \leq \nu-2\right\} \circ\left\{v_{q}: 2 \leq q \leq \nu\right\} \circ v_{1} w_{1} \circ\left\{x_{q} y_{q}: 1 \leq q \leq p-1\right\} \circ x_{p}=v^{\prime} .
\end{aligned}
$$

Subcase 3.2: $p=v$ :

$$
\begin{aligned}
P_{H}\left(u^{\prime}, v^{\prime}\right): u^{\prime}= & \left\{z_{q}: 1 \leq q \leq \nu\right\} \circ\left\{y_{v-q}: 0 \leq q \leq \nu-1\right\} \circ \\
& \left\{x_{q}: 1 \leq q \leq \nu-1\right\} \circ\left\{w_{\nu-q}: 1 \leq q \leq \nu-1\right\} \circ\left\{v_{q}: 1 \leq q \leq \nu\right\} \circ w_{\nu} x_{\nu}=v^{\prime} .
\end{aligned}
$$

Case 4: $u^{\prime}=z_{1}$ and $v^{\prime}=w_{p}, 1 \leq p \leq v$

Subcase 4.1: $1 \leq p \leq \nu-1$ :

$$
\begin{aligned}
P_{H}\left(u^{\prime}, v^{\prime}\right): u^{\prime}= & \left\{z_{q}: 1 \leq q \leq \nu\right\} \circ\left\{y_{\nu-q}: 0 \leq q \leq \nu-1\right\} \circ\left\{x_{q}: 1 \leq q \leq \nu\right\} \circ \\
& \left\{w_{\nu-q}: 0 \leq q \leq \nu-p-1\right\} \circ\left\{v_{q}: p+1 \leq q \leq \nu\right\} \circ\left\{v_{q} w_{q}: 1 \leq q \leq p\right\}=v^{\prime} .
\end{aligned}
$$

Subcase 4.2: $p=v$ : 


$$
\begin{aligned}
P_{H}\left(u^{\prime}, v^{\prime}\right): u^{\prime}= & \left\{z_{q}: 1 \leq q \leq \nu\right\} \circ\left\{y_{\nu-q}: 0 \leq q \leq \nu-1\right\} \circ x 1 \circ \\
& \left\{x_{\nu-q}: 0 \leq q \leq \nu-2\right\} \circ\left\{w_{q}: 2 \leq q \leq \nu-1\right\} \circ\left\{v_{\nu-q}: 0 \leq q \leq \nu-1\right\} \circ v_{1} w_{\nu}=v^{\prime} .
\end{aligned}
$$

Case 5: $u^{\prime}=z_{1}$ and $v^{\prime}=v_{p}, 1 \leq p \leq v$

$$
\begin{aligned}
P_{H}\left(u^{\prime}, v^{\prime}\right): u^{\prime}= & \left\{z_{q}: 1 \leq q \leq \nu\right\} \circ\left\{y_{\nu-q}: 0 \leq q \leq \nu-1\right\} \circ\left\{x_{q}: 1 \leq q \leq \nu\right\} \circ \\
& \left\{w_{\nu-q}: 0 \leq q \leq \nu-p\right\} \circ\left\{v_{q}: p+1 \leq q \leq \nu\right\} \circ\left\{v_{q} w_{q}: 1 \leq q \leq p-1\right\} \circ v_{p}=v^{\prime} .
\end{aligned}
$$

Case 6: $u^{\prime}=y_{1}$ and $v^{\prime}=z_{p}, 1 \leq p \leq v$

Subcase 6.1: $1 \leq p \leq \nu-1$ :

$$
\begin{aligned}
P_{H}\left(u^{\prime}, v^{\prime}\right): u^{\prime}= & y_{1} x_{1} w_{1} \circ\left\{v_{q}: 1 \leq q \leq \nu\right\} \circ\left\{w_{\nu-q}: 0 \leq q \leq \nu-2\right\} \circ\left\{x_{q} y_{q}: 2 \leq q \leq p\right\} \circ \\
& \left\{x_{q}: p+1 \leq q \leq \nu\right\} \circ\left\{y_{v-q}: 0 \leq q \leq \nu-p-1\right\} \circ\left\{z_{q}: p+1 \leq q \leq \nu\right\} \circ\left\{z_{q}: 1 \leq q \leq p\right\}=v^{\prime} .
\end{aligned}
$$

Subcase 6.2: $p=v$ :

$$
\begin{aligned}
P_{H}\left(u^{\prime}, v^{\prime}\right): u^{\prime}= & y_{1} \circ\left\{z_{q}: 1 \leq q \leq \nu-1\right\} \circ\left\{y_{v-q}: 1 \leq q \leq \nu-2\right\} \circ \\
& \left\{x_{q}: 2 \leq q \leq \nu\right\} \circ\left\{w_{v-q}: 0 \leq q \leq \nu-2\right\} \circ\left\{v_{q}: 2 \leq q \leq \nu\right\} \circ v_{1} w_{1} x_{1} y_{\nu} z_{\nu}=v^{\prime} .
\end{aligned}
$$

Case 7: $u^{\prime}=y_{1}$ and $v^{\prime}=y_{p}, 2 \leq p \leq v$

Subcase 8.1: $2 \leq p \leq \nu-1$ :

$$
\begin{aligned}
P_{H}\left(u^{\prime}, v^{\prime}\right): u^{\prime}= & y_{1} \circ\left\{z_{q}: 1 \leq q \leq \nu\right\} \circ\left\{y_{\nu-q}: 0 \leq q \leq \nu-p-1\right\} \circ\left\{x_{q}: p+1 \leq q \leq \nu\right\} \circ \\
& \left\{w_{\nu-q}: 0 \leq q \leq \nu-2\right\} \circ\left\{v_{q}: 2 \leq q \leq \nu\right\} \circ v_{1} w_{1} x_{1} \circ\left\{x_{q} y_{q}: 2 \leq q \leq p\right\}=v^{\prime} .
\end{aligned}
$$

Subcase 8.2: $p=v$ :

$$
\begin{aligned}
P_{H}\left(u^{\prime}, v^{\prime}\right): u^{\prime}= & y_{1} z_{1} \circ\left\{z_{\nu-q}: 0 \leq q \leq \nu-2\right\} \circ\left\{y_{q}: 2 \leq q \leq v-1\right\} \circ \\
& \left\{x_{\nu-q}: 1 \leq q \leq \nu-1\right\} \circ\left\{w_{q}: 1 \leq q \leq \nu-1\right\} \circ\left\{v_{\nu-q}: 1 \leq q \leq \nu-1\right\} \circ v_{\nu} w_{\nu} x_{\nu} y_{\nu}=v^{\prime} .
\end{aligned}
$$

Case 8: $u^{\prime}=y_{1}$ and $v^{\prime}=x_{p}, 1 \leq p \leq v$

Subcase 8.1: $p=1$ :

$$
\begin{aligned}
P_{H}\left(u^{\prime}, v^{\prime}\right): u^{\prime}= & y_{1} z_{1} \circ\left\{z_{v-q}: 0 \leq q \leq \nu-2\right\} \circ\left\{y_{q}: 2 \leq q \leq \nu\right\} \circ \\
& \left\{x_{v-q}: 0 \leq q \leq v-2\right\} \circ\left\{w_{q}: 2 \leq q \leq \nu\right\} \circ\left\{v_{\nu-q}: 0 \leq q \leq \nu-2\right\} \circ v_{1} w_{1} x_{1}=v^{\prime} .
\end{aligned}
$$

Subcase 8.2: $2 \leq p \leq v-1$ : 


$$
\begin{aligned}
P_{H}\left(u^{\prime}, v^{\prime}\right): u^{\prime}= & y_{1} \circ\left\{z_{q}: 1 \leq q \leq \nu\right\} \circ\left\{y_{v-q}: 0 \leq q \leq \nu-p\right\} \circ\left\{x_{q}: p+1 \leq q \leq \nu\right\} \circ \\
& \left\{w_{\nu-q}: 0 \leq q \leq v-2\right\} \circ\left\{v_{q}: 2 \leq q \leq \nu\right\} \circ v_{1} w_{1} x_{1} \circ\left\{x_{q} y_{q}: 2 \leq q \leq p-1\right\} \circ x_{p}=v^{\prime} .
\end{aligned}
$$

Subcase 8.3: $p=v$ :

$$
\begin{aligned}
P_{H}\left(u^{\prime}, v^{\prime}\right): u^{\prime}= & y_{1} z_{1} \circ\left\{z_{\nu-q}: 0 \leq q \leq v-2\right\} \circ\left\{y_{q}: 2 \leq q \leq v\right\} \circ \\
& \left\{x_{q}: 1 \leq q \leq v-1\right\} \circ\left\{w_{v-q}: 1 \leq q \leq v-1\right\} \circ\left\{v_{q}: 1 \leq q \leq v\right\} \circ w_{v} x_{v}=v^{\prime} .
\end{aligned}
$$

Case 9: $u^{\prime}=y_{1}$ and $v^{\prime}=w_{p}, 1 \leq p \leq v$

Subcase 9.1: $1 \leq p \leq \nu-1$ :

$$
\begin{aligned}
P_{H}\left(u^{\prime}, v^{\prime}\right): u^{\prime}= & y_{1} z_{1} \circ\left\{z_{v-q}: 0 \leq q \leq v-2\right\} \circ\left\{y_{q}: 2 \leq q \leq \nu\right\} \circ\left\{x_{q}: 1 \leq q \leq \nu\right\} \circ \\
& \left\{w_{v-q}: 0 \leq q \leq v-p-1\right\} \circ\left\{v_{q}: p+1 \leq q \leq \nu\right\} \circ\left\{v_{q} w_{q}: 1 \leq q \leq p\right\}=v^{\prime} .
\end{aligned}
$$

Subcase 9.2: $p=v$ :

$$
\begin{aligned}
P_{H}\left(u^{\prime}, v^{\prime}\right): u^{\prime}= & y_{1} z_{1} \circ\left\{z_{\nu-q}: 0 \leq q \leq v-2\right\} \circ\left\{y_{q}: 2 \leq q \leq v\right\} \circ\left\{x_{v-q}: 0 \leq q \leq v-1\right\} \circ \\
& \left\{w_{q}: 1 \leq q \leq v-1\right\} \circ\left\{v_{v-q}: 1 \leq q \leq v-1\right\} \circ v_{\nu} w_{v}=v^{\prime} .
\end{aligned}
$$

Case 10: $u^{\prime}=y_{1}$ and $v^{\prime}=v_{p}, 1 \leq p \leq v$

Subcase 10.1: $1 \leq p \leq v-1$ :

$$
\begin{aligned}
P_{H}\left(u^{\prime}, v^{\prime}\right): u^{\prime}= & y_{1} z_{1} \circ\left\{z_{v-q}: 0 \leq q \leq v-2\right\} \circ\left\{y_{q}: 2 \leq q \leq v\right\} \circ\left\{x_{q}: 1 \leq q \leq v\right\} \circ \\
& \left\{w_{v-q}: 0 \leq q \leq v-p\right\} \circ\left\{v_{q}: p+1 \leq q \leq v\right\} \circ v_{1} \circ\left\{w_{q} v_{q+1}: 1 \leq q \leq p-1\right\}=v^{\prime} .
\end{aligned}
$$

Subcase 10.2: $p=v$ :

$$
\begin{aligned}
P_{H}\left(u^{\prime}, v^{\prime}\right): u^{\prime}= & y_{1} z_{1} \circ\left\{z_{v-q}: 0 \leq q \leq v-2\right\} \circ\left\{y_{q}: 2 \leq q \leq v\right\} \circ\left\{x_{q}: 1 \leq q \leq v\right\} \circ \\
& \left\{w_{v-q}: 0 \leq q \leq v-1\right\} \circ\left\{v_{q}: 1 \leq q \leq v\right\}=v^{\prime} .
\end{aligned}
$$

Case 11: $u^{\prime}=x_{1}$ and $v^{\prime}=z_{p}, 1 \leq p \leq v$

Subcase 11.1: $1 \leq p \leq v-1$ :

$$
\begin{aligned}
P_{H}\left(u^{\prime}, v^{\prime}\right): u^{\prime}= & x_{1} w_{1} v_{1} \circ\left\{v_{v-q}: 0 \leq q \leq v-2\right\} \circ\left\{w_{q}: 2 \leq q \leq v\right\} \circ\left\{x_{v-q}: 0 \leq q \leq v-p-1\right\} \circ \\
& \left\{y_{p-q} x_{p-q}: 0 \leq q \leq p-2\right\} \circ y_{1} \circ\left\{y_{v-q}: 0 \leq q \leq v-p-1\right\} \circ\left\{z_{q}: p+1 \leq q \leq v\right\} \circ\left\{z_{q}: 1 \leq q \leq p\right\}=v^{\prime} .
\end{aligned}
$$

Subcase 11.2: $p=v$ : 


$$
\begin{aligned}
P_{H}\left(u^{\prime}, v^{\prime}\right): u^{\prime}= & \left\{x_{q}: 1 \leq q \leq v-1\right\} \circ\left\{w_{\nu-q}: 1 \leq q \leq \nu-1\right\} \circ\left\{v_{q}: 1 \leq q \leq \nu\right\} \circ \\
& w_{\nu} x_{\nu} \circ\left\{y_{\nu-q}: 0 \leq q \leq \nu-1\right\} \circ\left\{z_{q}: 1 \leq q \leq \nu\right\}=v^{\prime} .
\end{aligned}
$$

Case 12: $u^{\prime}=x_{1}$ and $v^{\prime}=y_{p}, 2 \leq p \leq v$

Subcase 12.1: $1 \leq p \leq \nu-1$ :

$$
\begin{aligned}
P_{H}\left(u^{\prime}, v^{\prime}\right): u^{\prime}= & x_{1} w_{1} v_{1} \circ\left\{v_{q} w_{q}: 1 \leq q \leq \nu\right\} \circ\left\{x_{\nu-q}: 0 \leq q \leq \nu-p-1\right\} \circ\left\{y_{q}: p+1 \leq q \leq \nu\right\} \circ \\
& \left\{z_{v-q}: 0 \leq q \leq \nu-1\right\} \circ y_{1} \circ\left\{x_{q} v_{q}: 2 \leq q \leq p\right\}=v^{\prime} .
\end{aligned}
$$

Subcase 12.2: $p=v$ :

$$
\begin{aligned}
P_{H}\left(u^{\prime}, v^{\prime}\right): u^{\prime}= & x_{1} w_{1} v_{1} \circ\left\{v_{q} w_{q}: 2 \leq q \leq \nu\right\} \circ\left\{x_{\nu-q}: 0 \leq q \leq \nu-2\right\} \circ\left\{y_{q}: 1 \leq q \leq v-1\right\} \circ \\
& \left\{z_{v-q}: 1 \leq q \leq v-1\right\} \circ z_{\nu} y_{\nu}=v^{\prime} .
\end{aligned}
$$

Case 13: $u^{\prime}=x_{1}$ and $v^{\prime}=x_{p}, 2 \leq p \leq \nu$

Subcase 13.1: $2 \leq p \leq \nu-1$ :

$$
\begin{aligned}
P_{H}\left(u^{\prime}, v^{\prime}\right): u^{\prime}= & x_{1} y_{1} \circ\left\{z_{q}: 1 \leq q \leq \nu\right\} \circ\left\{y_{\nu-q}: 0 \leq q \leq \nu-p\right\} \circ\left\{y_{p-q} x_{p-q}: 1 \leq q \leq p-1\right\} \circ \\
& \left\{x_{\nu-q}: 0 \leq q \leq \nu-p-1\right\} \circ\left\{w_{q}: p+1 \leq q \leq \nu\right\} \circ\left\{v_{\nu-q}: 0 \leq q \leq \nu-1\right\} \circ\left\{w_{q}: 1 \leq q \leq p\right\} \circ x_{p}=v^{\prime} .
\end{aligned}
$$

Subcase 13.2: $p=v$ :

$$
\begin{aligned}
P_{H}\left(u^{\prime}, v^{\prime}\right): u^{\prime}= & x_{1} y_{1} \circ\left\{z_{q}: 1 \leq q \leq \nu\right\} \circ\left\{y_{\nu-q}: 0 \leq q \leq \nu-2\right\} \circ\left\{x_{q}: 2 \leq q \leq \nu-1\right\} \circ \\
& \left\{w_{\nu-q}: 1 \leq q \leq \nu-1\right\} \circ\left\{w_{q}: 1 \leq q \leq \nu\right\} \circ w_{\nu} x_{\nu}=v^{\prime} .
\end{aligned}
$$

Case 14: $u^{\prime}=x_{1}$ and $v^{\prime}=w_{p}, 1 \leq p \leq v$

Subcase 14.1: $1 \leq p \leq \nu-1$ :

$$
\begin{aligned}
P_{H}\left(u^{\prime}, v^{\prime}\right): u^{\prime}= & x_{1} y_{1} \circ\left\{z_{q}: 1 \leq q \leq \nu\right\} \circ\left\{y_{\nu-q}: 0 \leq q \leq \nu-2\right\} \circ\left\{x_{q}: 2 \leq q \leq \nu\right\} \circ \\
& \left\{w_{\nu-q}: 0 \leq q \leq \nu-p-1\right\} \circ\left\{v_{q}: p+1 \leq q \leq \nu\right\} \circ\left\{v_{q} w_{q}: 1 \leq q \leq p\right\}=v^{\prime} .
\end{aligned}
$$

Subcase 14.2: $p=v$ :

$$
\begin{aligned}
P_{H}\left(u^{\prime}, v^{\prime}\right): u^{\prime}= & x_{1} y_{1} z_{1} \circ\left\{z_{\nu-q}: 0 \leq q \leq v-2\right\} \circ\left\{y_{q}: 2 \leq q \leq \nu\right\} \circ\left\{x_{\nu-q}: 0 \leq q \leq v-2\right\} \circ \\
& \left\{w_{q}: 2 \leq q \leq v-1\right\} \circ\left\{v_{\nu-q}: 0 \leq q \leq v-1\right\} \circ w_{1} w_{\nu}=v^{\prime} .
\end{aligned}
$$

Case 15: $u^{\prime}=x_{1}$ and $v^{\prime}=v_{p}, 1 \leq p \leq v$

Subcase 15.1: $1 \leq p \leq \nu-1$ : 


$$
\begin{aligned}
P_{H}\left(u^{\prime}, v^{\prime}\right): u^{\prime}= & x_{1} y_{1} \circ\left\{z_{q}: 1 \leq q \leq \nu\right\} \circ\left\{y_{v-q}: 0 \leq q \leq \nu-2\right\} \circ\left\{x_{q}: 2 \leq q \leq \nu\right\} \circ \\
& \left\{w_{v-q}: 0 \leq q \leq \nu-p\right\} \circ\left\{v_{q}: p+1 \leq q \leq \nu\right\} \circ\left\{v_{q} w_{q}: 1 \leq q \leq p-1\right\} \circ v_{p}=v^{\prime} .
\end{aligned}
$$

Subcase 15.2: $p=v$ :

$$
\begin{aligned}
P_{H}\left(u^{\prime}, v^{\prime}\right): u^{\prime}= & x_{1} y_{1} \circ\left\{z_{q}: 1 \leq q \leq \nu\right\} \circ\left\{y_{\nu-q}: 0 \leq q \leq \nu-2\right\} \circ\left\{x_{q}: 2 \leq q \leq \nu\right\} \circ \\
& \left\{w_{\nu-q}: 0 \leq q \leq \nu-1\right\} \circ\left\{v_{q}: 1 \leq q \leq \nu\right\}=v^{\prime} .
\end{aligned}
$$

Case 16: $u^{\prime}=w_{1}$ and $v^{\prime}=z_{p}, 1 \leq p \leq v$

Subcase 16.1: $1 \leq p \leq \nu-1$ :

$$
\begin{aligned}
P_{H}\left(u^{\prime}, v^{\prime}\right): u^{\prime}= & w_{1} v_{1} \circ\left\{v_{\nu-q}: 0 \leq q \leq \nu-2\right\} \circ\left\{w_{q}: 2 \leq q \leq \nu\right\} \circ\left\{x_{v-q}: 0 \leq q \leq \nu-p\right\} \circ \\
& \left\{y_{q}: p \leq q \leq \nu\right\} \circ\left\{x_{q} y_{q}: 1 \leq q \leq p-1\right\} \circ\left\{z_{p-q}: 1 \leq q \leq p-1\right\} \circ\left\{z_{v-q}: 0 \leq q \leq v-p\right\}=v^{\prime} .
\end{aligned}
$$

Subcase 16.2: $p=v$ :

$$
\begin{aligned}
P_{H}\left(u^{\prime}, v^{\prime}\right): u^{\prime}= & w_{1} \circ\left\{v_{q}: 1 \leq q \leq \nu\right\} \circ\left\{w_{\nu-q}: 0 \leq q \leq \nu-2\right\} \circ\left\{x_{q}: 2 \leq q \leq \nu\right\} \circ \\
& x_{1} \circ\left\{y_{v-q}: 0 \leq q \leq \nu-1\right\} \circ\left\{z_{q}: 1 \leq q \leq \nu\right\}=v^{\prime} .
\end{aligned}
$$

Case 17: $u^{\prime}=w_{1}$ and $v^{\prime}=y_{p}, 1 \leq p \leq v$

Subcase 17.1: $1 \leq p \leq \nu-1$ :

$$
\begin{aligned}
P_{H}\left(u^{\prime}, v^{\prime}\right): u^{\prime}= & w_{1} v_{1} \circ\left\{v_{v-q}: 0 \leq q \leq \nu-2\right\} \circ\left\{w_{q}: 2 \leq q \leq \nu\right\} \circ\left\{x_{\nu-q}: 0 \leq q \leq v-p\right\} \circ \\
& \left\{y_{p-q} x_{p-q}: 1 \leq q \leq p-1\right\} \circ\left\{y_{v-q}: 0 \leq q \leq \nu-p-1\right\} \circ\left\{z_{q}: p+1 \leq q \leq \nu\right\} \circ\left\{z_{q}: 1 \leq q \leq p\right\} \circ y_{p}=v^{\prime} .
\end{aligned}
$$

Subcase 17.2: $p=v$ :

$$
\begin{gathered}
P_{H}\left(u^{\prime}, v^{\prime}\right): u^{\prime}=w_{1} v_{1} \circ\left\{v_{v-q}: 0 \leq q \leq \nu-2\right\} \circ\left\{w_{q}: 2 \leq q \leq \nu\right\} \circ\left\{x_{\nu-q}: 0 \leq q \leq \nu-1\right\} \circ \\
\\
\left\{y_{v-q}: 0 \leq q \leq \nu-1\right\} \circ\left\{z_{q}: 1 \leq q \leq \nu\right\}=v^{\prime} .
\end{gathered}
$$

Case 18: $u^{\prime}=w_{1}$ and $v^{\prime}=x_{p}, 1 \leq p \leq v$

Subcase 18.1: $1 \leq p \leq \nu-1$ :

$$
\begin{aligned}
P_{H}\left(u^{\prime}, v^{\prime}\right): u^{\prime}= & w_{1} v_{1} \circ\left\{v_{\nu-q}: 0 \leq q \leq \nu-2\right\} \circ\left\{w_{q}: 2 \leq q \leq \nu\right\} \circ\left\{x_{\nu-q}: 0 \leq q \leq v-p-1\right\} \circ \\
& \left\{y_{q}: p+1 \leq q \leq v-1\right\} \circ\left\{z_{\nu-q}: 1 \leq q \leq v-1\right\} \circ z_{\nu} y_{\nu} \circ\left\{x_{q} y_{q}: 1 \leq q \leq p-1\right\} \circ x_{p}=v^{\prime} .
\end{aligned}
$$

Subcase 18.2: $p=v$ : 


$$
\begin{aligned}
P_{H}\left(u^{\prime}, v^{\prime}\right): u^{\prime}= & w_{1} \circ\left\{v_{q}: 1 \leq q \leq \nu\right\} \circ\left\{w_{\nu-q}: 0 \leq q \leq v-2\right\} \circ\left\{x_{q}: 2 \leq q \leq v-1\right\} \circ \\
& \left\{y_{v-q}: 1 \leq q \leq \nu-1\right\} \circ\left\{z_{q}: 1 \leq q \leq \nu\right\} \circ y_{\nu} x_{1} x_{v}=v^{\prime} .
\end{aligned}
$$

Case 19: $u^{\prime}=w_{1}$ and $v^{\prime}=w_{p}, 2 \leq p \leq v$

Subcase 19.1: $2 \leq p \leq v-1$ :

$$
\begin{aligned}
P_{H}\left(u^{\prime}, v^{\prime}\right): u^{\prime}= & w_{1} x_{1} y_{1} \circ\left\{z_{q}: 1 \leq q \leq \nu\right\} \circ\left\{y_{\nu-q}: 0 \leq q \leq \nu-2\right\} \circ\left\{x_{q}: 2 \leq q \leq \nu\right\} \circ \\
& \left\{w_{\nu-q}: 0 \leq q \leq \nu-p-1\right\} \circ\left\{v_{q}: p+1 \leq q \leq \nu\right\} \circ\left\{v_{q} w_{q}: 1 \leq q \leq p\right\}=v^{\prime} .
\end{aligned}
$$

Subcase 19.2: $p=v$ :

$$
\begin{aligned}
P_{H}\left(u^{\prime}, v^{\prime}\right): u^{\prime}= & w_{1} x_{1} y_{1} z_{1} \circ\left\{z_{\nu-q}: 0 \leq q \leq v-2\right\} \circ\left\{y_{q}: 2 \leq q \leq \nu\right\} \circ\left\{x_{\nu-q}: 0 \leq q \leq v-2\right\} \circ \\
& \left\{w_{q}: 2 \leq q \leq v-1\right\} \circ\left\{v_{v-q}: 0 \leq q \leq v-1\right\} \circ v_{\nu} w_{v}=v^{\prime} .
\end{aligned}
$$

Case 20: $u^{\prime}=w_{1}$ and $v^{\prime}=v_{p}, 1 \leq p \leq v$

Subcase 20.1: $p=1$ :

$$
\begin{aligned}
P_{H}\left(u^{\prime}, v^{\prime}\right): u^{\prime}= & w_{1} x_{1} y_{1} \circ\left\{z_{q}: 1 \leq q \leq \nu\right\} \circ\left\{y_{\nu-q}: 0 \leq q \leq \nu-2\right\} \circ\left\{x_{q}: 2 \leq q \leq \nu\right\} \circ \\
& \left\{w_{\nu-q}: 0 \leq q \leq \nu-2\right\} \circ\left\{v_{q}: 2 \leq q \leq \nu\right\} \circ v_{1}=v^{\prime} .
\end{aligned}
$$

Subcase 20.2: $2 \leq p \leq v-1$ :

$$
\begin{aligned}
P_{H}\left(u^{\prime}, v^{\prime}\right): u^{\prime}= & w_{1} x_{1} y_{1} \circ\left\{z_{q}: 1 \leq q \leq \nu\right\} \circ\left\{y_{v-q}: 0 \leq q \leq \nu-2\right\} \circ\left\{x_{q}: 2 \leq q \leq \nu\right\} \circ \\
& \left\{w_{\nu-q}: 0 \leq q \leq \nu-p\right\} \circ\left\{v_{q}: p+1 \leq q \leq \nu\right\} \circ\left\{v_{q} w_{q}: 1 \leq q \leq p-1\right\} \circ v_{p}=v^{\prime} .
\end{aligned}
$$

Subcase 20.3: $p=v$ :

$$
\begin{aligned}
P_{H}\left(u^{\prime}, v^{\prime}\right): u^{\prime}= & w_{1} \circ\left\{v_{q}: 1 \leq q \leq v-1\right\} \circ\left\{w_{\nu-q}: 0 \leq q \leq v-2\right\} \circ\left\{x_{q}: 2 \leq q \leq v-1\right\} \circ \\
& \left\{y_{\nu-q}: 1 \leq q \leq v-1\right\} \circ\left\{z_{q}: 1 \leq q \leq \nu\right\} \circ y_{\nu} x_{1} x_{\nu} w_{\nu} v_{v}=v^{\prime} .
\end{aligned}
$$

Case 21: $u^{\prime}=v_{1}$ and $v^{\prime}=z_{p}, 1 \leq p \leq v$

Subcase 21.1: $p=1$ :

$$
\begin{aligned}
P_{H}\left(u^{\prime}, v^{\prime}\right): u^{\prime}= & \left\{v_{q}: 1 \leq q \leq \nu\right\} \circ\left\{w_{\nu-q}: 0 \leq q \leq \nu-1\right\} \circ x_{1} \circ\left\{x_{\nu-q}: 0 \leq q \leq \nu-2\right\} \circ \\
& \left\{w_{q}: 1 \leq q \leq \nu\right\} \circ\left\{z_{\nu-q}: 0 \leq q \leq \nu-1\right\}=v^{\prime} .
\end{aligned}
$$

Subcase 21.2: $2 \leq p \leq v-1$ : 


$$
\begin{aligned}
P_{H}\left(u^{\prime}, v^{\prime}\right): u^{\prime}= & \left\{v_{q}: 1 \leq q \leq \nu\right\} \circ\left\{w_{\nu-q}: 0 \leq q \leq \nu-1\right\} \circ\left\{x_{q} y_{q}: 1 \leq q \leq p-2\right\} \circ\left\{x_{q}: p-1 \leq q \leq \nu\right\} \circ \\
& \left\{y_{\nu-q}: 0 \leq q \leq \nu-p+1\right\} \circ\left\{z_{p-q}: 1 \leq q \leq p-1\right\} \circ\left\{z_{\nu-q}: 0 \leq q \leq \nu-p\right\}=v^{\prime} .
\end{aligned}
$$

Subcase 21.3: $p=v$ :

$$
\begin{aligned}
P_{H}\left(u^{\prime}, v^{\prime}\right): u^{\prime}= & \left\{v_{q}: 1 \leq q \leq \nu\right\} \circ\left\{w_{\nu-q}: 0 \leq q \leq \nu-1\right\} \circ\left\{x_{q}: 1 \leq q \leq \nu\right\} \circ \\
& \left\{y_{\nu-q}: 0 \leq q \leq \nu-1\right\} \circ\left\{z_{q}: 1 \leq q \leq \nu\right\}=v^{\prime} .
\end{aligned}
$$

Case 22: $u^{\prime}=v_{1}$ and $v^{\prime}=y_{p}, 1 \leq p \leq v$

Subcase 22.1: $1 \leq p \leq \nu-1$ :

$$
\begin{aligned}
P_{H}\left(u^{\prime}, v^{\prime}\right): u^{\prime}= & \left\{v_{q}: 1 \leq q \leq \nu\right\} \circ\left\{w_{\nu-q}: 0 \leq q \leq \nu-1\right\} \circ\left\{x_{q} y_{q}: 1 \leq q \leq p-1\right\} \circ\left\{x_{q}: p \leq q \leq \nu\right\} \circ \\
& \left\{y_{v-q}: 0 \leq q \leq \nu-p-1\right\} \circ\left\{z_{q}: p+1 \leq q \leq \nu\right\} \circ\left\{z_{q}: 1 \leq q \leq p\right\}=v^{\prime} .
\end{aligned}
$$

Subcase 22.2: $p=v$ :

$$
\begin{aligned}
P_{H}\left(u^{\prime}, v^{\prime}\right): u^{\prime}= & \left\{v_{q}: 1 \leq q \leq \nu\right\} \circ\left\{w_{\nu-q}: 0 \leq q \leq \nu-1\right\} \circ\left\{x_{\nu-q}: 0 \leq q \leq \nu-1\right\} \circ \\
& \left\{y_{q}: 1 \leq q \leq \nu-1\right\} \circ\left\{z_{v-q}: 1 \leq q \leq \nu-1\right\} \circ z_{\nu} y_{v}=v^{\prime} .
\end{aligned}
$$

Case 23: $u^{\prime}=v_{1}$ and $v^{\prime}=x_{p}, 1 \leq p \leq v$

Subcase 23.1: $1 \leq p \leq \nu-1$ :

$$
\begin{aligned}
P_{H}\left(u^{\prime}, v^{\prime}\right): u^{\prime}= & v_{1} \circ\left\{v_{v-q}: 0 \leq q \leq \nu-2\right\} \circ\left\{w_{q}: 1 \leq q \leq \nu\right\} \circ\left\{x_{\nu-q}: 0 \leq q \leq v-p-1\right\} \circ \\
& \left\{y_{q}: p \leq q \leq v-1\right\} \circ\left\{z_{v-q}: 1 \leq q \leq \nu-1\right\} \circ z_{\nu} y_{\nu} \circ\left\{x_{q} y_{q}: 1 \leq q \leq p-1\right\} \circ x_{p}=v^{\prime} .
\end{aligned}
$$

Subcase 23.2: $p=v$ :

$$
\begin{aligned}
P_{H}\left(u^{\prime}, v^{\prime}\right): u^{\prime}= & \left\{v_{q}: 1 \leq q \leq \nu\right\} \circ\left\{w_{v-q}: 0 \leq q \leq v-1\right\} \circ\left\{x_{q}: 1 \leq q \leq \nu-1\right\} \circ \\
& \left\{y_{v-q}: 1 \leq q \leq v-1\right\} \circ\left\{z_{q}: 1 \leq q \leq \nu\right\} \circ y_{\nu} x_{v}=v^{\prime} .
\end{aligned}
$$

Case 24: $u^{\prime}=v_{1}$ and $v^{\prime}=w_{p}, 1 \leq p \leq v$

Subcase 24.1: $1 \leq p \leq \nu-1$ :

$$
\begin{aligned}
P_{H}\left(u^{\prime}, v^{\prime}\right): u^{\prime}= & \left\{v_{q} w_{q}: 1 \leq q \leq p-1\right\} \circ\left\{v_{q}: p \leq q \leq \nu\right\} \circ\left\{w_{\nu-q}: 0 \leq q \leq \nu-p-1\right\} \circ \\
& \left\{x_{q}: p+1 \leq q \leq \nu\right\} \circ\left\{y_{v-q}: 0 \leq q \leq \nu-2\right\} \circ\left\{z_{q}: 2 \leq q \leq \nu\right\} \circ z_{1} y_{1} \circ\left\{x_{q}: 1 \leq q \leq p\right\} \circ w_{p}=v^{\prime} .
\end{aligned}
$$

Subcase 24.2: $p=v$ : 


$$
\begin{gathered}
P_{H}\left(u^{\prime}, v^{\prime}\right): u^{\prime}=v_{1} \circ\left\{v_{v-q}: 0 \leq q \leq v-2\right\} \circ\left\{w_{q}: 1 \leq q \leq v-1\right\} \circ\left\{x_{v-q}: 0 \leq q \leq v-1\right\} \circ \\
\\
\left\{y_{q}: 1 \leq q \leq v-1\right\} \circ\left\{z_{v-q}: 1 \leq q \leq v-1\right\} \circ z_{\nu} y_{v} x_{v} w_{v}=v^{\prime} .
\end{gathered}
$$

Case 25: $u^{\prime}=v_{1}$ and $v^{\prime}=v_{p}, 2 \leq p \leq v$

Subcase 25.1: $2 \leq p \leq v-1$ :

$$
\begin{aligned}
P_{H}\left(u^{\prime}, v^{\prime}\right): u^{\prime}= & v_{1} w_{1} x_{1} y_{1} \circ\left\{z_{q}: 1 \leq q \leq v\right\} \circ\left\{y_{\nu-q}: 0 \leq q \leq v-2\right\} \circ\left\{x_{q}: 2 \leq q \leq v\right\} \circ \\
& \left\{w_{\nu-q} v_{v-q}: 0 \leq q \leq v-p-1\right\} \circ\left\{w_{p-q}: 0 \leq q \leq p-2\right\} \circ\left\{v_{q}: 2 \leq q \leq p\right\}=v^{\prime} .
\end{aligned}
$$

Subcase 25.2: $p=v$ :

$$
\begin{aligned}
P_{H}\left(u^{\prime}, v^{\prime}\right): u^{\prime}= & v_{1} w_{1} x_{1} y_{1} \circ\left\{z_{q}: 1 \leq q \leq v\right\} \circ\left\{y_{v-q}: 0 \leq q \leq \nu-2\right\} \circ\left\{x_{q}: 2 \leq q \leq \nu\right\} \circ \\
& \left\{w_{\nu-q}: 0 \leq q \leq v-2\right\} \circ\left\{v_{q}: 2 \leq q \leq v\right\}=v^{\prime} .
\end{aligned}
$$

The existence of the Hamiltonian path between every pair of vertices of the $H_{v}$ completes the proof.

Using Theorems 1 and 2, the following proposition computes the detour index of $H_{v}$.

Corollary 1. Let $G=H_{v}$, where $v \geq 4$. Then, the detour index of $G$ is

$$
\omega(G)=\frac{5 v(5 v-1)^{2}}{2} .
$$

Proof. The number of vertices in the graph $G$ is $5 \nu$. Replacing $5 v$ with $n$ in Theorem 1 gives the proposition.

\section{Hamilton-Connectivity and the Detour Index of $G_{v}$}

In this section, we show that the graph $G_{v}$ is Hamiltonconnected. Afterwards, we use the Hamilton-connectivity to find the analytical exact expression of the detour index of the graph $G_{v}$.

The vertex set of $G_{v}$ consists of four layers of vertices, i.e., $w_{p}, x_{p}, y_{p}$, and $z_{p}$. That is to say that $V\left(G_{v}\right)=$ $\left\{w_{p}, x_{p}, y_{p}, z_{p}: 1 \leq p \leq v\right\}$. Accordingly, the edge set of $G_{v}$ is as follows:

$$
\begin{aligned}
E\left(G_{v}\right)= & \left\{w_{p} w_{p+1}, x_{p} x_{p+1}, y_{p} y_{p+1}, z_{p} z_{p+1}, w_{p} x_{p}, x_{p} y_{p},\right. \\
& \left.y_{p} x_{p+1}, y_{p} z_{p}: 1 \leq p \leq v-1\right\} .
\end{aligned}
$$

The subscripts are to be considered modulo $v$. Figure 3 presents the $\nu$-dimensional convex polytope $G_{v}$ with proper labeling of vertices which will be used to show its Hamiltonconnectivity.

The following is the main result of this section.

Theorem 3. The graph $v$-dimensional convex polytope $G_{\nu}$, with $v \geq 5$, is Hamilton-connected.

Proof. We prove this result by definition. For this, we have to show that there exist Hamiltonian paths between any pair of vertices of $G_{\gamma}$.

Let $P_{H}(u, v)$ be a Hamiltonian path between vertices $u$ and $v$ in $G_{v}$. Let $G_{v}=Z \cup Y \cup X \cup W$ such that $Z=\left\{z_{1}, z_{2}, \ldots, z_{\nu}\right\}, \quad Y=\left\{y_{1}, y_{2}, \ldots, y_{\nu}\right\}, \quad X=\left\{x_{1}, x_{2}, \ldots\right.$, $\left.x_{\nu}\right\}$, and $W=\left\{w_{1}, w_{2}, \ldots, w_{\nu}\right\}$ (see Figure 3).

Case 1: $u=z_{1}$ and $v=z_{p}, 2 \leq p \leq v$

Subcase 1.1: $2 \leq p \leq v-2$ :

$$
\begin{aligned}
P_{H}(u, v): u= & z_{1} \circ\left\{z_{v-q}: 0 \leq q \leq v-p-1\right\} \circ\left\{y_{p-q+1}: 0 \leq q \leq p-2\right\} \circ\left\{x_{q}: 3 \leq q \leq p+1\right\} \circ \\
& \left\{x_{q} y_{q}: p+2 \leq q \leq \nu\right\} \circ y_{1} x_{1} w_{1} \circ\left\{w_{\nu-q}: 0 \leq q \leq v-2\right\} \circ x_{2} y_{2} \circ\left\{z_{q}: 2 \leq q \leq p\right\}=v .
\end{aligned}
$$

Subcase 1.2: $p=v-1$ : 


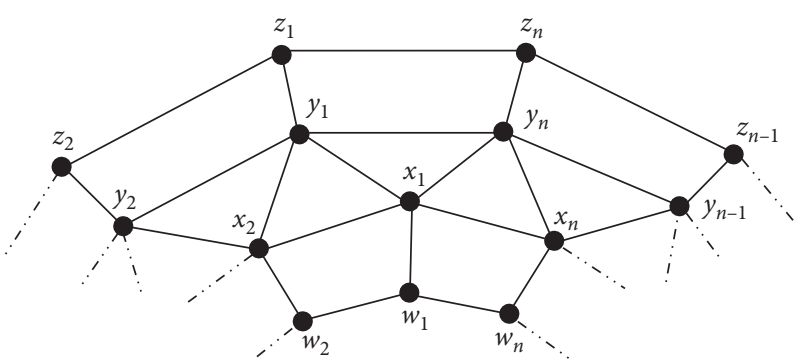

Figure 3: The $v$-dimensional convex polytope $G_{\nu}$.

$$
\begin{aligned}
P_{H}(u, v): u= & z_{1} z_{\nu} \circ\left\{y_{v-q}: 0 \leq q \leq v-3\right\} \circ\left\{x_{q}: 3 \leq q \leq \nu\right\} \circ\left\{w_{\nu-q}: 0 \leq q \leq v-1\right\} \circ \\
& x_{1} y_{1} x_{2} y_{2} \circ\left\{z_{q}: 2 \leq q \leq v-1\right\}=v .
\end{aligned}
$$

Subcase 1.3: $p=v$ :

$$
\begin{aligned}
P_{H}(u, v): u= & z_{1} y_{1} x_{1} w_{1} \circ\left\{w_{\nu-q}: 0 \leq q \leq \nu-2\right\} \circ\left\{x_{q}: 2 \leq q \leq \nu\right\} \circ \\
& \left\{y_{\nu-q}: 0 \leq q \leq \nu-2\right\} \circ\left\{z_{q}: 2 \leq q \leq \nu\right\}=v .
\end{aligned}
$$

Case 2: $u=z_{1}$ and $v=y_{p}, 1 \leq p \leq v$

Subcase 2.1: $1 \leq p \leq \nu-1$ :

$$
\begin{aligned}
P_{H}(u, v): u= & \left\{z_{q}: 1 \leq q \leq \nu\right\} \circ\left\{y_{\nu-q}: 0 \leq q \leq \nu-p-1\right\} \circ\left\{x_{q}: p+1 \leq q \leq \nu\right\} \circ \\
& \left\{w_{\nu-q}: 0 \leq q \leq \nu-1\right\} \circ\left\{x_{q} y_{q}: 1 \leq q \leq p\right\}=v .
\end{aligned}
$$

Subcase 2.2: $p=v$ :

$$
\begin{aligned}
P_{H}(u, v): u= & z_{1} \circ\left\{z_{v-q}: 0 \leq q \leq v-2\right\} \circ\left\{y_{q}: 2 \leq q \leq v-1\right\} \circ\left\{x_{v-q}: 1 \leq q \leq v-2\right\} \circ \\
& y_{1} x_{1} \circ\left\{w_{q}: 1 \leq q \leq \nu\right\} \circ x_{v} y_{v}=v .
\end{aligned}
$$

Case 3: $u=z_{1}$ and $v=x_{p}, 1 \leq p \leq v$

Subcase 3.1: $1 \leq p \leq \nu-1$ :

$$
\begin{aligned}
P_{H}(u, v): u= & z_{1} \circ\left\{z_{q}: 2 \leq q \leq \nu\right\} \circ\left\{y_{v-q}: 0 \leq q \leq \nu-p\right\} \circ\left\{x_{q}: p+1 \leq q \leq \nu\right\} \circ \\
& \left\{w_{\nu-q}: 0 \leq q \leq \nu-1\right\} \circ\left\{x_{q} y_{q}: 2 \leq q \leq p-1\right\} \circ x_{p}=v .
\end{aligned}
$$

Subcase 3.2: $p=v$ :

$$
\begin{aligned}
P_{H}(u, v): u= & \left\{z_{q}: 1 \leq q \leq \nu\right\} \circ\left\{y_{\nu-q}: 0 \leq q \leq \nu-1\right\} \circ\left\{x_{q}: 1 \leq q \leq \nu-1\right\} \circ \\
& \left\{w_{\nu-q}: 1 \leq q \leq \nu-1\right\} \circ w_{\nu} x_{\nu}=v .
\end{aligned}
$$


Case 4: $u=z_{1}$ and $v=w_{p}, 1 \leq p \leq v$

Subcase 4.1: $p=1$ :

$$
\begin{aligned}
P_{H}(u, v): u= & \left\{z_{q}: 1 \leq q \leq \nu\right\} \circ\left\{y_{v-q}: 0 \leq q \leq v-1\right\} \\
& \circ\left\{x_{q}: 1 \leq q \leq \nu\right\} \circ \\
& \left\{w_{\nu-q}: 0 \leq q \leq v-1\right\}=v .
\end{aligned}
$$

$$
\begin{array}{r}
P_{H}(u, v): u=\left\{z_{q}: 1 \leq q \leq \nu\right\} \circ\left\{y_{\nu-q}: 0 \leq q \leq \nu-p+1\right\} \circ\left\{x_{q}: p \leq q \leq \nu\right\} \circ \\
\left\{x_{q} y_{q}: 1 \leq q \leq p-2\right\} \circ x_{p-1} \circ\left\{w_{p-q}: 1 \leq q \leq p-1\right\} \circ\left\{w_{\nu-q}: 0 \leq q \leq \nu-p\right\}=v .
\end{array}
$$

Case 5: $u=y_{1}$ and $v=z_{p}, 1 \leq p \leq v$ Subcase 5.1: $1 \leq p \leq v-1$ :

$$
\begin{aligned}
P_{H}(u, v): u= & y_{1} x_{1} w_{1} \circ\left\{w_{\nu-q}: 0 \leq q \leq v-2\right\} \circ\left\{x_{q} y_{q}: 2 \leq q \leq p\right\} \circ\left\{x_{q}: p+1 \leq q \leq v\right\} \circ \\
& \left\{y_{\nu-q}: 0 \leq q \leq v-p-1\right\} \circ\left\{z_{q}: p+1 \leq q \leq v\right\} \circ\left\{z_{q}: 1 \leq q \leq p\right\}=v .
\end{aligned}
$$

Subcase 5.2: $p=v$ :

$$
\begin{aligned}
P_{H}(u, v): u= & y_{1} \circ\left\{z_{q}: 1 \leq q \leq v-1\right\} \circ\left\{y_{v-q}: 1 \leq q \leq v-2\right\} \circ\left\{x_{q}: 2 \leq q \leq v-1\right\} \circ \\
& \left\{w_{v-q}: 1 \leq q \leq v-1\right\} \circ w_{v} x_{v} x_{1} y_{v} z_{v}=v .
\end{aligned}
$$

Case 6: $u=y_{1}$ and $v=y_{p}, 2 \leq p \leq v$

Subcase 6.1: $2 \leq p \leq v-1$ :

$$
\begin{aligned}
P_{H}(u, v): u= & y_{1} \circ\left\{z_{q}: 1 \leq q \leq v\right\} \circ\left\{y_{v-q}: 0 \leq q \leq v-p-1\right\} \circ\left\{x_{q}: p+1 \leq q \leq v\right\} \circ \\
& \left\{w_{v-q}: 0 \leq q \leq v-1\right\} \circ x_{1} \circ\left\{x_{q} y_{q}: 2 \leq q \leq p\right\}=v .
\end{aligned}
$$

Subcase 6.2: $p=v$ :

$$
\begin{aligned}
P_{H}(u, v): u= & y_{1} z_{1} \circ\left\{z_{v-q}: 0 \leq q \leq v-2\right\} \circ\left\{y_{q}: 2 \leq q \leq v-1\right\} \circ\left\{x_{v-q}: 1 \leq q \leq v-1\right\} \circ \\
& \left\{w_{q}: 1 \leq q \leq v\right\} \circ x_{v} y_{v}=v .
\end{aligned}
$$

Case 7: $u=y_{1}$ and $v=x_{p}, 1 \leq p \leq v$

Subcase 7.1: $p=1$ :

$$
\begin{aligned}
P_{H}(u, v): u= & y_{1} \circ\left\{z_{q}: 1 \leq q \leq v\right\} \circ\left\{y_{v-q}: 0 \leq q \leq v-2\right\} \circ\left\{x_{q}: 2 \leq q \leq v\right\} \circ \\
& \left\{w_{v-q}: 0 \leq q \leq v-1\right\} \circ x_{1}=v .
\end{aligned}
$$


Subcase 7.2: $2 \leq p \leq v-1$ :

$$
\begin{aligned}
P_{H}(u, v): u= & y_{1} \circ\left\{z_{q}: 1 \leq q \leq v\right\} \circ\left\{y_{v-q}: 0 \leq q \leq v-p\right\} \circ\left\{x_{q}: p+1 \leq q \leq v\right\} \circ \\
& \left\{w_{v-q}: 0 \leq q \leq v-1\right\} \circ x_{1} \circ\left\{x_{q} y_{q}: 2 \leq q \leq p-1\right\} \circ x_{p}=v .
\end{aligned}
$$

Subcase 7.3: $p=v$ :

$$
\begin{aligned}
P_{H}(u, v): u= & y_{1} z_{1} \circ\left\{z_{\nu-q}: 0 \leq q \leq v-2\right\} \circ\left\{y_{q}: 2 \leq q \leq v\right\} \circ\left\{x_{q}: 1 \leq q \leq v-1\right\} \circ \\
& \left\{w_{\nu-q}: 1 \leq q \leq v-1\right\} \circ w_{v} x_{v}=v .
\end{aligned}
$$

Case 8: $u=y_{1}$ and $v=w_{p}, 1 \leq p \leq v$

Subcase 8.1: $p=1$ :

$$
\begin{aligned}
P_{H}(u, v): u= & y_{1} z_{1} \circ\left\{z_{v-q}: 0 \leq q \leq v-2\right\} \circ\left\{y_{q}: 2 \leq q \leq v\right\} \circ\left\{x_{q}: 1 \leq q \leq v\right\} \circ \\
& \left\{w_{\nu-q}: 0 \leq q \leq v-1\right\}=v .
\end{aligned}
$$

Subcase $8.2: 2 \leq p \leq \nu-1$ :

$$
\begin{aligned}
P_{H}(u, v): u= & y_{1} x_{1} \circ\left\{x_{q} y_{q}: 2 \leq q \leq p\right\} \circ\left\{z_{p-q}: 0 \leq q \leq p-1\right\} \circ\left\{z_{\nu-q}: 0 \leq q \leq v-p-1\right\} \circ \\
& \left\{y_{q}: p+1 \leq q \leq v\right\} \circ\left\{x_{\nu-q}: 0 \leq q \leq v-p-1\right\} \circ\left\{w_{q}: p+1 \leq q \leq \nu\right\} \circ \\
& \left\{w_{q}: 1 \leq q \leq p\right\}=v .
\end{aligned}
$$

Subcase 8.3: $p=v$ :

$$
\begin{aligned}
P_{H}(u, v): u= & y_{1} \circ\left\{z_{q}: 1 \leq q \leq v\right\} \circ\left\{y_{\nu-q}: 0 \leq q \leq v-2\right\} \circ\left\{x_{q}: 2 \leq q \leq v\right\} \circ \\
& x_{1} \circ\left\{w_{q}: 1 \leq q \leq v\right\}=v .
\end{aligned}
$$

Case 9: $u=x_{1}$ and $v=z_{p}, 1 \leq p \leq v$

Subcase 9.1: $1 \leq p \leq \nu-1$ :

$P_{H}(u, v): u=x_{1} \circ\left\{w_{q}: 1 \leq q \leq \nu\right\} \circ\left\{x_{\nu-q}: 0 \leq q \leq v-p-1\right\} \circ$

$\left\{y_{p-q} x_{p-q}: 0 \leq q \leq p-2\right\} \circ y_{1}$

$\circ\left\{y_{v-q}: 0 \leq q \leq v-p-1\right\} 。$ $\left\{z_{q}: p+1 \leq q \leq \nu\right\} \circ\left\{z_{q}: 1 \leq q \leq p\right\}=v$.
Subcase 9.2: $p=v$ :

$$
\begin{aligned}
P_{H}(u, v): u= & x_{1} \circ\left\{w_{q}: 1 \leq q \leq \nu\right\} \circ\left\{x_{\nu-q}: 0 \leq q \leq v-2\right\} \circ \\
& \left\{y_{q}: 2 \leq q \leq v\right\} \circ y_{1} \circ\left\{z_{q}: 1 \leq q \leq v\right\}=v .
\end{aligned}
$$

Case 10: $u=x_{1}$ and $v=y_{p}, 1 \leq p \leq v$

Subcase 10.1: $1 \leq p \leq \nu-1$ : 


$$
\begin{gathered}
P_{H}(u, v): u=x_{1} \circ\left\{w_{q}: 1 \leq q \leq v\right\} \circ\left\{x_{v-q}: 0 \leq q \leq v-p-1\right\} \circ\left\{y_{q}: p+1 \leq q \leq v\right\} \circ \\
\left\{z_{v-q}: 0 \leq q \leq v-1\right\} \circ y_{1} \circ\left\{x_{q} y_{q}: 2 \leq q \leq p\right\}=v .
\end{gathered}
$$

Subcase 10.2: $p=v$ :

$$
\begin{aligned}
P_{H}(u, v): u= & x_{1} \circ\left\{w_{q}: 1 \leq q \leq v\right\} \circ\left\{x_{v-q}: 0 \leq q \leq v-2\right\} \circ\left\{y_{q}: 1 \leq q \leq v-1\right\} \circ \\
& \left\{z_{v-q}: 1 \leq q \leq v-1\right\} \circ z_{v} y_{v}=v .
\end{aligned}
$$

Case 11: $u=x_{1}$ and $v=x_{p}, 2 \leq p \leq v$

Subcase 11.1: $2 \leq p \leq v-1$ :

$$
\begin{gathered}
P_{H}(u, v): u=x_{1} \circ\left\{w_{q}: 1 \leq q \leq v\right\} \circ\left\{x_{v-q}: 0 \leq q \leq v-p-1\right\} \circ\left\{y_{q}: p \leq q \leq v\right\} \circ \\
\left\{z_{v-q}: 0 \leq q \leq v-1\right\} \circ\left\{y_{q-1} x_{q}: 2 \leq q \leq p\right\}=v .
\end{gathered}
$$

Subcase 11.2: $p=v$ :

$$
\begin{aligned}
P_{H}(u, v): u= & x_{1} w_{1} \circ\left\{w_{v-q}: 0 \leq q \leq v-2\right\} \circ\left\{x_{q}: 2 \leq q \leq v-1\right\} \circ\left\{y_{v-q}: 1 \leq q \leq v-1\right\} \circ \\
& \left\{z_{q}: 1 \leq q \leq v\right\} \circ y_{v} x_{v}=v .
\end{aligned}
$$

Case 12: $u=x_{1}$ and $v=w_{p}, 1 \leq p \leq v$

Subcase 12.2: $2 \leq p \leq v-1$ :

Subcase 12.1: $p=1$ :

$P_{H}(u, v): u=x_{1} y_{1} \circ\left\{z_{q}: 1 \leq q \leq v\right\} \circ\left\{y_{v-q}: 0 \leq q \leq v-2\right\} \circ$

$$
\left\{x_{q}: 2 \leq q \leq \nu\right\} \circ\left\{w_{\nu-q}: 0 \leq q \leq v-1\right\}=v .
$$

$$
\begin{aligned}
P_{H}(u, v): u= & x_{1} \circ\left\{y_{q-1} x_{q}: 2 \leq q \leq p-1\right\} \circ\left\{w_{p-q}: 1 \leq q \leq p-1\right\} \circ\left\{w_{v-q}: 0 \leq q \leq v-p-1\right\} \circ \\
& \left\{x_{q}: p+1 \leq q \leq v\right\} \circ\left\{y_{v-q}: 0 \leq q \leq v-p\right\} \circ\left\{z_{q}: p \leq q \leq v\right\} \circ \\
& \left\{z_{q}: 1 \leq q \leq p-1\right\} \circ y_{p-1} x_{p} w_{p}=v .
\end{aligned}
$$

Subcase 12.3: $p=v$ :

$$
\begin{aligned}
P_{H}(u, v): u= & x_{1} \circ\left\{w_{q}: 1 \leq q \leq v-1\right\} \circ\left\{x_{\nu-q}: 1 \leq q \leq v-2\right\} \circ\left\{y_{q}: 1 \leq q \leq v-1\right\} \circ \\
& \left\{z_{v-q}: 1 \leq q \leq v-1\right\} \circ z_{v} y_{v} x_{\nu} w_{v}=v .
\end{aligned}
$$

Case 13: $u=w_{1}$ and $v=z_{p}, 1 \leq p \leq v$

Subcase 13.1: $1 \leq p \leq v-1$ : 


$$
\begin{aligned}
P_{H}(u, v): u= & \left\{w_{q}: 1 \leq q \leq \nu\right\} \circ\left\{x_{\nu-q}: 0 \leq q \leq \nu-p-1\right\} \circ\left\{y_{p-q} x_{p-q}: 0 \leq q \leq p-1\right\} \circ \\
& \left\{y_{\nu-q}: 0 \leq q \leq \nu-p-1\right\} \circ\left\{z_{q}: p+1 \leq q \leq \nu\right\} \circ\left\{z_{q}: 1 \leq q \leq p\right\}=v .
\end{aligned}
$$

Subcase 13.2: $p=v$ :

$$
\begin{aligned}
P_{H}(u, v): u= & \left\{w_{q}: 1 \leq q \leq \nu\right\} \circ\left\{x_{\nu-q}: 0 \leq q \leq v-1\right\} \\
& \circ\left\{y_{v-q}: 0 \leq q \leq \nu-1\right\} \circ \\
& \left\{z_{q}: 1 \leq q \leq v\right\}=v .
\end{aligned}
$$

Case 14: $u=w_{1}$ and $v=y_{p}, 1 \leq p \leq \nu$

Subcase 14.1: $1 \leq p \leq \nu-1$ :

$$
\begin{aligned}
P_{H}(u, v): u= & \left\{w_{q}: 1 \leq q \leq \nu\right\} \circ\left\{x_{\nu-q}: 0 \leq q \leq \nu-p\right\} \circ\left\{y_{p-q} x_{p-q}: 1 \leq q \leq p-1\right\} \circ \\
& \left\{y_{\nu-q}: 0 \leq q \leq \nu-p-1\right\} \circ\left\{z_{q}: p+1 \leq q \leq \nu\right\} \circ\left\{z_{q}: 1 \leq q \leq p\right\} \circ y_{p}=v .
\end{aligned}
$$

Subcase 14.2: $p=v$ :

$$
\begin{aligned}
P_{H}(u, v): u= & \left\{w_{q}: 1 \leq q \leq \nu\right\} \circ\left\{x_{\nu-q}: 0 \leq q \leq \nu-1\right\} \\
& \circ\left\{y_{q}: 1 \leq q \leq \nu-1\right\} \circ \\
& \left\{z_{v-q}: 1 \leq q \leq \nu-1\right\} \circ z_{\nu} y_{v}=v .
\end{aligned}
$$

Subcase 15.1: $p=1$ :

$$
\begin{aligned}
P_{H}(u, v): u= & \left\{w_{q}: 1 \leq q \leq \nu\right\} \circ\left\{x_{\nu-q}: 0 \leq q \leq \nu-2\right\} \\
& \circ\left\{y_{q}: 2 \leq q \leq \nu\right\} \circ \\
& \left\{z_{\nu-q}: 0 \leq q \leq \nu-1\right\} \circ y_{1} x_{1}=v .
\end{aligned}
$$

Case 15: $u=w_{1}$ and $v=x_{p}, 1 \leq p \leq v$

Subcase 15.2: $2 \leq p \leq \nu-1$ :

$$
\begin{aligned}
P_{H}(u, v): u= & \left\{w_{q}: 1 \leq q \leq \nu\right\} \circ\left\{x_{v-q}: 0 \leq q \leq v-p-1\right\} \circ\left\{y_{q}: p \leq q \leq \nu\right\} \circ \\
& \left\{z_{v-q}: 0 \leq q \leq \nu-1\right\} \circ y_{1} x_{1} \circ\left\{x_{q} y_{q}: 2 \leq q \leq p-1\right\} \circ x_{p}=v .
\end{aligned}
$$

Subcase 15.3: $p=v$ :

$P_{H}(u, v): u=w_{1} \circ\left\{w_{v-q}: 0 \leq q \leq \nu-2\right\}$

$\circ\left\{x_{q}: 2 \leq q \leq v-1\right\} \circ\left\{y_{v-q}: 1 \leq q \leq v-1\right\} \circ$

$\left\{z_{q}: 1 \leq q \leq \nu\right\} \circ y_{\nu} x_{1} x_{\nu}=v$.
Case 16: $u=w_{1}$ and $v=w_{p}, 2 \leq p \leq v$

Subcase 16.1: $2 \leq p \leq \nu-1$ :

$$
\begin{aligned}
P_{H}(u, v): u= & w_{1} \circ\left\{w_{\nu-q}: 0 \leq q \leq v-p-1\right\} \circ\left\{x_{q} y_{q}: p+1 \leq q \leq \nu\right\} \circ x_{1} y_{1} z_{1} \circ \\
& \left\{z_{\nu-q}: 0 \leq q \leq v-2\right\} \circ\left\{y_{q}: 2 \leq q \leq p\right\} \circ\left\{x_{p-q}: 0 \leq q \leq p-2\right\} \circ \\
& \left\{w_{q}: 2 \leq q \leq p\right\}=v .
\end{aligned}
$$

Subcase 16.2: $p=v$ : 


$$
\begin{aligned}
P_{H}(u, v): u= & \left\{w_{q}: 1 \leq q \leq v-1\right\} \circ\left\{x_{\nu-q}: 1 \leq q \leq v-1\right\} \\
& \circ\left\{y_{q}: 1 \leq q \leq v-1\right\} \circ \\
& \left\{z_{\nu-q}: 1 \leq q \leq v-1\right\} \circ z_{\nu} y_{\nu} x_{\nu} w_{\nu}=v .
\end{aligned}
$$

The existence of the Hamiltonian path between every pair of vertices of the $G_{v}$ completes the proof.

Using Theorems 1 and 3, the following proposition computes the detour index of $G_{v}$.

Corollary 2. Let $G=G_{v}$, where $v \geq 4$. Then, the detour index of $G$ is

$$
\omega(G)=\frac{4 \nu(4 v-1)^{2}}{2} .
$$

Proof. The number of vertices in the graph $G$ is $4 \nu$. Replacing $4 v$ with $n$ in Theorem 1 gives the proposition.

\section{Conclusions and Future Work}

Computing the detour index of a graph is NP-complete and checking if a graph is Hamilton-connected is also NPcomplete. In this paper, we construct three infinite families of Hamilton-connected convex polytope networks. Furthermore, we construct an infinite family of non-Hamiltonconnected convex polytope networks. The later construction shows that not all convex polytope networks are Hamiltonconnected. More importantly, we compute exact analytical expressions for the detour index of the families of Hamiltonconnected convex polytope networks.

In view of the work by Alspach and Liu [41], we propose the following conjectures [41]:

\section{Conjecture 1.}

(i) The generalized Petersen graph $G P(\nu, 4) \nu \geq 9$ is nonbipartite Hamilton-connected

(ii) The generalized Petersen graph $G P(\nu, 5) \nu \geq 11$ is nonbipartite Hamilton-connected if $\nu \mid 2$ and bipartite Hamilton-laceable if $\nu \nmid 2$

\section{Data Availability}

There are no data associated with the manuscript.

\section{Conflicts of Interest}

The authors declare that there are no conflicts of interest.

\section{References}

[1] O. Ore, "Hamilton-connected graphs," Journal of Pure and Applied Algebra, vol. 42, pp. 21-27, 1963.

[2] R. Frucht, "A canonical representation of trivalent Hamiltonian graphs," Journal of Graph Theory, vol. 1, pp. 45-60, 1976.
[3] V. S. Gordon, Y. L. Orlovich, and F. Werner, "Hamiltonian properties of triangular grid graphs," Discrete Mathematics, vol. 308, no. 24, pp. 6166-6188, 2008.

[4] S. Qiang, Z. Qain, and A. Yahui, "The Hamiltonicity of generalized honeycomb torus networks," Information Processing Letters, vol. 115, no. 2, pp. 104-111, 2005.

[5] I. A. Stewart, "Sufficient conditions for Hamiltonicity in multiswapped networks," Journal of Parallel and Distributed Computing, vol. 101, pp. 17-26, 2017.

[6] B. Wei, "Hamiltonian paths and Hamiltonian connectivity in graphs," Discrete Mathematics, vol. 121, no. 1-3, pp. 223-228, 1993.

[7] X. Yang, D. J. Evans, H. Lai, and G. M. Megson, "Generalized honeycomb torus is Hamiltonian," Information Processing Letters, vol. 92, no. 1, pp. 31-37, 2004.

[8] M. Bača, "Face anti-magic labelings of convex polytopes," Utilitas Mathematica, vol. 55, pp. 221-226, 1999.

[9] M. Baca, "Labelings of two classes of convex polytopes," Utilitas Mathematica, vol. 34, pp. 24-31, 1988.

[10] M. Bača, "On magic labellings of convex polytopes," Annals of Discrete Mathematics, vol. 51, pp. 13-16, 1992.

[11] M. Miller, M. Baca, and J. A. MacDougall, "Vertex-magic total labeling of generalized Petersen graphs and convex polytopes," Journal of Combinatorial Mathematics and Combinatorial Computing, vol. 59, pp. 89-99, 2006.

[12] M. Imran, A. Q. Baig, and A. Ahmad, "Families of plane graphs with constant metric dimension," Utilitas Mathematica, vol. 88, pp. 43-57, 2012.

[13] M. Imran, S. A. U. H. Bokhary, and A. Q. Baig, "On the metric dimension of rotationally-symmetric convex polytopes," Journal of Algebra Combinatorics Discrete Structures and Applications, vol. 3, pp. 45-59, 2015.

[14] M. Imran and H. M. A. Siddiqui, "Computing the metric dimension of convex polytopes generated by wheel related graphs," Acta Mathematica Hungarica, vol. 149, no. 1, pp. 10-30, 2016.

[15] M. Imran, S. A. Ul Haq Bokhary, and A. Q. Baig, "On families of convex polytopes with constant metric dimension," Computers \& Mathematics with Applications, vol. 60, no. 9, pp. 2629-2638, 2010.

[16] M. A. Malik and M. Sarwar, "On the metric dimension of two families of convex polytopes," Afrika Matematika, vol. 27, no. $1-2$, pp. 229-238, 2016.

[17] M. Imran, S. A. U. H. Bokhary, A. Ahmad, and A. Semaničová-feňovčíková, "On classes of regular graphs with constant metric dimension," Acta Mathematica Scientia, vol. 33, no. 1, pp. 187-206, 2013.

[18] J. Kratica, V. Kovačević-Vujčić, M. Čangalović, and M. Stojanović, "Minimal doubly resolving sets and the strong metric dimension of some convex polytopes," Applied Mathematics and Computation, vol. 218, no. 19, pp. 97909801, 2012.

[19] H. Raza, S. Hayat, and X.-F. Pan, "On the fault-tolerant metric dimension of convex polytopes," Applied Mathematics and Computation, vol. 339, pp. 172-185, 2018.

[20] H. Raza, J.-B. Liu, and S. Qu, "On mixed metric dimension of rotationally symmetric graphs," IEEE Access, vol. 8, pp. 11560-11569, 2020.

[21] A. Simić, M. Bogdanović, and J. Milošević, "The binary locating-dominating number of some convex polytopes," Ars Mathematica Contemporanea, vol. 13, pp. 367-377, 2017.

[22] H. Raza, S. Hayat, and X.-F. Pan, "Binary locating-dominating sets in rotationally-symmetric convex polytopes," Symmetry, vol. 10, no. 12, pp. 727-745, 2018. 
[23] A. L. J. Savić, Z. L. J. Maksimović, and M. S. Bogdanović, “The open-locating-dominating number of some convex polytopes," Filomat, vol. 32, no. 2, pp. 635-642, 2018.

[24] S. Hayat, A. Khan, S. Khan, and J.-B. Liu, "Hamilton connectivity of convex polytopes with applications to their detour index," Complexity, vol. 2021, Article ID 6684784, , 2021.

[25] I. Lukovits, "The detour index," Croatica Chemica Acta, vol. 69 , pp. 873-882, 1996.

[26] I. Lukovits, "Indicators for atoms included in cycles," Journal of Chemical Information and Computer Sciences, vol. 36, no. 1, pp. 65-68, 1996.

[27] N. Trinajstić, S. Nikolić, B. Lučić, D. Amić, and Z. Mihalić, "The detour matrix in chemistry," Journal for Chemical Information and Computer Scientists, vol. 37, pp. 631-638, 1997.

[28] G. Rücker and C. Rücker, "Symmetry-aided computation of the detour matrix and the detour index," Journal of Chemical Information and Computer Sciences, vol. 38, no. 4, pp. 710714, 1998.

[29] F. Harary, Graph Theory, p. 203, Addison-Wesley, Reading, MA, USA, 1969.

[30] A. Mahmiani, O. Khormali, and A. Iranmanesh, "The edge versions of detour index," MATCH Communications in Mathematical and in Computer Chemistry, vol. 62, no. 2, pp. 419-431, 2009.

[31] B. Zhou and X. Cai, "Index," Contesting Aging and Loss, vol. 63, pp. 199-210, 2010.

[32] X. Qi and B. Zhou, "Detour index of a class of unicyclic graphs," Filomat, vol. 24, no. 1, pp. 29-40, 2010.

[33] C. Du, "Minimum detour index of bicyclic graphs," MATCH Communications in Mathematical and in Computer Chemistry, vol. 68, no. 1, pp. 357-370, 2012.

[34] W. Fang, Z. Q. Cai, and X. X. Li, "Minimum detour index of tricyclic graphs," Journal of Chemistry, vol. 2019, Article ID 6031568, 8 pages, 2019.

[35] A. Karbasioun, A. R. Ashrafi, and M. V. Diudea, "Distance and detour matrices of an infinite class of dendrimer nanostars," MATCH Communications in Mathematical and in Computer Chemistry, vol. 63, no. 1, pp. 239-246, 2010.

[36] R. Wu and H. Deng, "Graphene quantum dots," Carbon Nanomaterials Sourcebook, vol. 8, no. 2, pp. 45-82, 2016.

[37] V. Kaladevi and A. Abinayaa, "On detour distance Laplacian energy," Journal of Informatics and Mathematical Sciences, vol. 9, no. 3, pp. 721-732, 2017.

[38] H. O. Abdullah and Z. I. Omar, "Edge restricted detour index of some graphs," Journal of Discrete Mathematical Sciences and Cryptography, vol. 23, no. 4, pp. 861-877, 2020.

[39] J.-H. Tang, U. Ali, M. Javaid, and K. Shabbir, "Zagreb connection indices of subdivision and semi-total point operations on graphs," Journal of Chemistry, vol. 2019, Article ID 9846913, 14 pages, 2019.

[40] A. A. Dobrynin, R. Entringer, and I. Gutman, "Wiener index of trees: theory and applications," Acta Applicandae Mathematicae, vol. 66, no. 3, pp. 211-249, 2001.

[41] B. Alspach and J. Liu, "On the Hamilton connectivity of generalized Petersen graphs," Discrete Mathematics, vol. 309, no. 17 , pp. 5461-5473, 2009. 